Interaction vertex imaging (IVI) for carbon ion therapy monitoring: a feasibility study

This content has been downloaded from IOPscience. Please scroll down to see the full text.

2012 Phys. Med. Biol. 574655

(http://iopscience.iop.org/0031-9155/57/14/4655)

View the table of contents for this issue, or go to the journal homepage for more

Download details:

IP Address: 132.239.1.231

This content was downloaded on 18/06/2017 at 12:21

Please note that terms and conditions apply.

You may also be interested in:

Absolute prompt-gamma yield measurements for ion beam therapy monitoring

M Pinto, M Bajard, S Brons et al.

Nuclear physics in particle therapy: a review

Marco Durante and Harald Paganetti

Measurement of charged particle yields from PMMA irradiated by a $220 \mathrm{MeV} / \mathrm{u} 12 \mathrm{C}$ beam

L Piersanti, F Bellini, F Bini et al.

Assessment and improvements of Geant4 hadronic models in the context of prompt-gamma hadrontherapy monitoring

G Dedes, M Pinto, D Dauvergne et al.

Fragmentation of 120 and $200 \mathrm{MeV}$ u1 $4 \mathrm{He}$ ions in water and PMMA targets

M Rovituso, C Schuy, U Weber et al.

Prompt gamma imaging with a slit camera for real-time range control in proton therapy

$\mathrm{J}$ Smeets, $\mathrm{F}$ Roellinghoff, $\mathrm{D}$ Prieels et al.

Ion beam transport in tissue-like media using MC code SHIELD-HIT

Irena Gudowska, Nikolai Sobolevsky, Pedro Andreo et al.

Experimental study of nuclear fragmentation of 200 and $400 \mathrm{MeV} / \mathrm{u} 12 \mathrm{C}$ ions in water for applications in particle therapy

E Haettner, $\mathrm{H}$ Iwase, $M$ Krämer et al. 


\title{
Interaction vertex imaging (IVI) for carbon ion therapy monitoring: a feasibility study
}

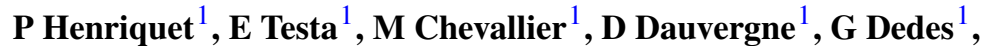 \\ N Freud ${ }^{2}$, J Krimmer ${ }^{1}$, J M Létang ${ }^{2}$, C Ray ${ }^{1}$, M-H Richard ${ }^{1,2}$ \\ and F Sauli ${ }^{3}$ \\ ${ }^{1}$ Institut de Physique Nucléaire de Lyon; Université de Lyon, F-69003 Villeurbanne, France; \\ IN2P3/CNRS, UMR 5822; Université de Lyon 1, F-69622 Villeurbanne, France \\ ${ }^{2}$ Université de Lyon, CREATIS; CNRS UMR 5220; Inserm U 1044; INSA-Lyon; \\ Université Lyon 1; Centre Léon Bérard, Lyon, France \\ 3 TERA Foundation, Novara, Italy \\ E-mail: e.testa@ipnl.in2p3.fr
}

Received 20 December 2011, in final form 18 April 2012

Published 2 July 2012

Online at stacks.iop.org/PMB/57/4655

\begin{abstract}
Proton imaging can be seen as a powerful technique for online monitoring of ion range during carbon ion therapy irradiations. Indeed, a large number of secondary protons are created during nuclear reactions, and many of these protons are likely to escape from the patient even for deep-seated tumors, carrying accurate information on the reaction vertex position. Two detection techniques have been considered: (i) double-proton detection by means of two forward-located trackers and (ii) single-proton detection in coincidence with the incoming carbon ion detected by means of a beam hodoscope. Geant 4 simulations, validated by proton yield measurements performed at GANIL and GSI, show that ion-range monitoring is accessible on a pencil-beam basis with the single-proton imaging technique. Millimetric precision on the Bragg peak position is expected in the ideal case of homogeneous targets. The uncertainties in more realistic conditions should be investigated, in particular the influence of tissue heterogeneity in the very last part of the ion path (about $20 \mathrm{~mm}$ ).
\end{abstract}

(Some figures may appear in colour only in the online journal)

\section{Introduction}

Online monitoring of the dose during treatments is one of the major challenges for the quality assurance of hadrontherapy. The ballistic precision of carbon ions, together with their large biological effectiveness at the end of their path in tissues, makes it possible to conform the dose to the tumor with high accuracy. Moreover, carbon ion therapy proves 
to be efficient for some radioresistant tumors, while sparing almost completely healthy tissues located downstream, alongside the ion beam and, to a lesser extent, in front of the tumor. However, slight deviations from the treatment plan may have dramatic consequences on the treatment outcome. Such deviations may arise from patient mispositioning, organ motion, changes in either tumor or healthy tissue volumes during the treatment period and from uncertainties in the stoichiometric calibration of the $\mathrm{x}$-ray $\mathrm{CT}$ image used for planning.

So far, the only way to check online that the dose is deposited at the right place is to take advantage of nuclear reactions, although they are not directly associated with dose deposition (governed by electromagnetic interaction processes). Some of the nuclear reactions give rise to the production of radioactive isotopes $\left({ }^{11} \mathrm{C},{ }^{10} \mathrm{C}\right.$ and $\left.{ }^{15} \mathrm{O}\right)$ which decay by means of positron emission. In the case of carbon ion therapy, the carbon isotopes are mainly concentrated close to the Bragg peak region. They allow for positron emission tomography (PET), which was used online at the GSI test treatment facility until 2008 (Enghardt et al 2004). Other sources of secondary radiation originating from nuclear reactions are envisaged for online imaging. Prompt gamma rays are intensively studied as their emission profile was shown to be correlated with the ion range in matter for both protons (Min et al 2006) and carbon ions (Testa et al 2008). Moreover, their fairly large multiplicity, together with their quasi-instantaneous emission, makes it possible to consider real-time monitoring of the ion range during proton or carbon treatments (Testa et al 2010, Moteabbed et al 2011). This would represent a major advance in the quality assurance as compared to PET for which the lifetimes of the radioactive isotopes delay the information.

In this work, we explore a third modality for online control of the ion range that has been first proposed by Amaldi et al (2010). This method called interaction vertex imaging (IVI) is based on the detection of secondary protons to reconstruct nuclear emission vertices. In the case of carbon ion therapy, it has been shown that a large number of protons are generated during nuclear collisions along the primary ion path (Gunzert-Marx et al 2008). As protons originating from the projectile nuclei have a most probable velocity and emission direction close to the incident ion direction, they are likely to escape from the patient and to be detected with high efficiency by means of a particle detector located downstream from the patient. The aim is finally to provide a means of controlling the ion range by comparing the distributions of reconstructed vertices calculated in the treatment planning stage and the distributions measured during the irradiation. This comparison of measured and simulated distributions is also performed during the control procedure of PET devices used at GSI or HIT where predicted and measured $\beta^{+}$distributions are compared to detect potential deviations from the treatment plan (Enghardt et al 2004). In the case of active beam delivery, 'real-time' ion-range control can be defined as the monitoring of the range of each single pencil beam (raster position).

We present the results of a preliminary study which investigates the potentiality of this method to determine in real time the Bragg peak location. Two techniques have been considered: double-proton interaction vertex imaging (DP-IVI, coincidence between two protons emitted from the same vertex) and single-proton interaction vertex imaging (SP-IVI, the vertex is reconstructed using the incident carbon ion trajectory, determined by a beam hodoscope). Section 2 presents the IVI principles, our simulation tool (Geant4 9.2) and its validation against experimental proton yields and finally the scope of this work. Results are then discussed, in particular regarding the spatial resolution that can be expected on a pencil-beam basis with IVI. 

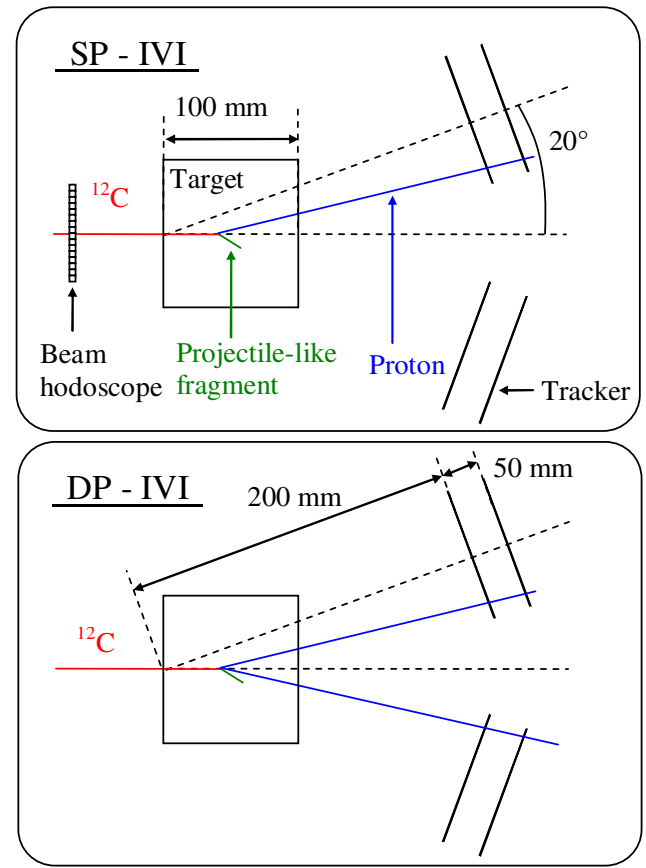

Figure 1. Principle of proton IVI and description of the setup used in our simulation. Upper diagram: single-proton imaging (SP-IVI): only one trajectory is required, provided the incident transverse position of the carbon ion beam is determined, by means of a beam hodoscope. Lower diagram: double-proton imaging (DP-IVI): two protons are emitted from the same interaction vertex. Two sets of tracking detectors are used to reconstruct the intersection point of the trajectories. Target: PMMA cylinder $10 \mathrm{~cm}$ in length and diameter used for the comparison of SP-IVI and DP-IVI. Tracker: pair of $50 \mu \mathrm{m}$ thick silicon detectors of $10 \times 10 \mathrm{~cm}^{2}$.

\section{Materials and methods}

\subsection{IVI principles and the common features of the simulated setups}

Interaction vertex reconstruction is a core issue in high-energy experiments. For hadrontherapy control purposes, it can consist in determining the intersection of two trajectories assumed to be straight lines. Two detection techniques have been considered, and are presented in figure 1: (i) double-proton detection (DP-IVI) by means of two forward-located detectors (called 'trackers'), and (ii) single-proton detection, in coincidence with the incoming carbon ion detected by means of a beam hodoscope (SP-IVI). Although double-proton detection necessarily leads to lower detection statistics, it was still considered because it requires a simpler setup (without beam hodoscope).

For this initial feasibility study, a simple reconstruction algorithm is sufficient. The vertex is defined as the point at minimum and equal distances between the two straight lines of the proton and incident ion trajectories (SP-IVI) or the two proton trajectories (DP-IVI). More sophisticated algorithms using most likely trajectories will probably lead to higher image quality, once the principles of the method are well established.

The basic features of the simulated setups correspond to figure 1: a PMMA target and two trackers placed $20 \mathrm{~cm}$ from the target entrance and at $\theta=20^{\circ}$ with respect to the beam 
direction. Trackers consist of two pairs of $10 \mathrm{~cm} \times 10 \mathrm{~cm} \times 50 \mu \mathrm{m}$ silicon detectors. The distance between the two silicon detectors is $5 \mathrm{~cm}$. The spatial resolution of these detectors was conservatively considered to be equal to the pixel size although we know that it can be far smaller, considering the pixellated CMOS detectors currently available at IPHC-Strasbourg (Baudot et al 2009).

The beam hodoscope developed in our laboratory is composed of square scintillating fibers $1 \mathrm{~mm}$ in width. Since nuclear reactions induced in this detector are negligible, they are not modeled in the simulations. As a matter of fact, a set of two hodoscopes is in principle required to obtain the incidence direction of the beam, but it can be assumed that, sufficiently close to the patient, the transverse position of the beam provides enough information. On the whole, the geometrical parameters of the setup are not crucial, apart from the angular position of the trackers: as secondary protons are emitted in the forward direction, this angular position is a trade-off between statistics and precision of vertex reconstruction (the latter requires large angles for sharper intersection of trajectories). It was set to $20^{\circ}$ in order to optimize vertex reconstruction precision.

\subsection{The Monte Carlo simulation tool and its validation against experimental data}

Monte Carlo simulations have been increasingly used in the field of ion beam therapy in recent years. The most popular Monte Carlo packages are FLUKA (Fassò et al 2005), Geant4 (Apostolakis et al 2009), SHIELD-HIT (Gudowska et al 2004) and PHITS (Iwase et al 2002). Studies using such codes can be classified into the following topics: macro- (Pshenichnov et al 2008) and micro- (Villagrasa et al 2011) dosimetry and nuclear imaging for hadrontherapy monitoring (Moteabbed et al 2011) (references are examples of studies among many others using the Geant4 toolkit like this work).

We have chosen the Geant4 toolkit (version 9.2) whose high configurability allows users to choose either to assemble a physics list in a process-by-process approach or, alternatively, to use pre-defined partial or complete lists, which are already included in the Geant 4 package. The complete user-defined physics list applied in this study can call two different nuclear reaction models, namely the binary cascade (BC) and the quantum molecular dynamics (QMD) models. This makes it possible to evaluate the influence of model uncertainties on the energy and angular spectra of secondary protons. Unless otherwise mentioned, the results shown in this paper have been obtained with the QMD model which in general provides a better description of the emission yields at beam energies commonly used in hadrontherapy (Böhlen et al 2010).

Both electromagnetic and hadronic physics models are regularly updated and improved in Geant4. Regarding the present feasibility study, two quantities are of particular interest: the yield of secondary protons emerging from the target and proton straggling that is the main source of uncertainty in reconstructed vertex locations. According to the conclusions of previous works (Pshenichnov et al 2010, Grevillot et al 2010), we can consider that Geant4 9.2 predictions of such quantities are satisfactory for a feasibility study. Nevertheless, to be fully confident in our simulations, we performed our own validation of the properties of secondary protons.

We focus here on the validation of the secondary proton yields: such yields have been measured in the framework of this study at high energy $\left(310 \mathrm{MeV} \mathrm{u}^{-1}\right)$ to complete experimental data obtained at GSI (Gunzert-Marx et al 2008) with $200 \mathrm{MeV} \mathrm{u}^{-1}{ }^{12} \mathrm{C}$ beam and a $128 \mathrm{~mm}$ thick water target and at GANIL (Braunn et al 2011) with $95 \mathrm{MeV} \mathrm{u}^{-112} \mathrm{C}$ beam and PMMA targets of various thicknesses (the comparison with simulations is done with the $25 \mathrm{~mm}$ target). 

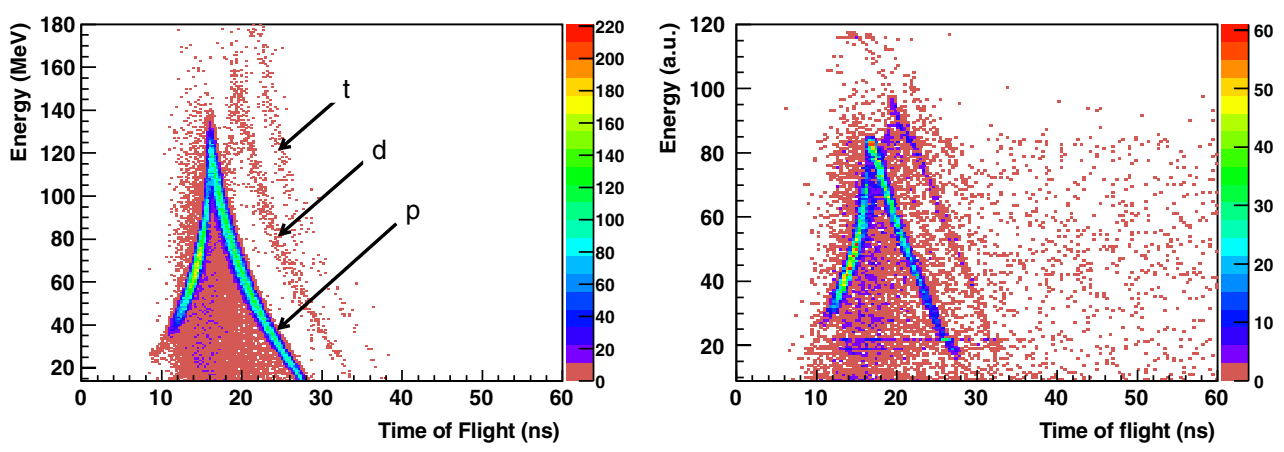

Figure 2. Energy versus time of flight (expressed in nanoseconds) distributions obtained for $310 \mathrm{MeV} \mathrm{u}^{-1}$ carbon ions incident on a $21 \mathrm{~cm}$ thick water target with the telescope located at $30^{\circ}$ with respect to the beam direction and $2.2 \mathrm{~m}$ from the target center. Left: simulation, right: measurements (GSI experiment). Protons, deuterons and tritons are referred to as $p, d$ and $t$ in the simulated distributions (the background events are mainly due to fragmentation reactions in the scintillator).

We used a $310 \mathrm{MeV} \mathrm{u}^{-1}{ }^{12} \mathrm{C}$ ion beam irradiating a $21 \mathrm{~cm}$ thick water target. A single telescope was successively placed at $30^{\circ}$ and $45^{\circ}$ with respect to the beam and in the forward direction, at a distance of $2.2 \mathrm{~m}$ from the target center. This telescope consisted of a thin plastic scintillator followed by a $\mathrm{NaI}(\mathrm{Tl})$ scintillator cylinder $5 \mathrm{~cm}$ in diameter and $5 \mathrm{~cm}$ in length. Another thin scintillator was set upstream from the target to allow time of flight (TOF) measurements, triggered by incident ions. The TOF of the detected particle, together with the energy deposited in the $\mathrm{NaI}(\mathrm{Tl})$ detector, allowed us to identify particles, namely protons, deuterons and tritons (figure 2). The triangular shape of the distributions corresponding to each particle is due to the fact that high-energy particles (with TOF lower than $17 \mathrm{~ns}$ ) cross the scintillator and deposit only a fraction of their energy. The energy scale (vertical axis) corresponds to the equivalent proton energy deposition: the maximum energy deposited by protons in the $5 \mathrm{~cm}$ long $\mathrm{NaI}$ scintillator (upper point of the triangular shape distribution) was set to the corresponding energy deposition calculated by SRIM (Ziegler et al 2010).

Taking into account the solid angle of the telescope, we report in figure 3 the measured and simulated values of the detected proton yields as a function of detection angle for the three beam energies $\left(95,200\right.$ and $\left.310 \mathrm{MeV} \mathrm{u}^{-1}\right)$. Apart from small angles $\left(<5^{\circ}\right)$, simulations overestimate the secondary proton yields at 200 and $310 \mathrm{MeV} \mathrm{u}^{-1}$ : this overestimation ranges from $10 \%$ to $40 \%$ at $200 \mathrm{MeV} \mathrm{u}^{-1}$ and from $30 \%$ to $50 \%$ at $310 \mathrm{MeV} \mathrm{u}^{-1}$ (the percentages are related to experimental values). At $95 \mathrm{MeV} \mathrm{u}^{-1}$, the simulations lead to secondary proton yields lower than experimental yields: $20 \%$ at $16^{\circ}$ and $87 \%$ at $40^{\circ}$ (for the QMD model). Nevertheless, the overall agreement was considered satisfactory enough to carry out this feasibility study using Geant4 9.2, especially with trackers placed at $20^{\circ}$ with respect to the beam direction.

\subsection{The scope of the feasibility study}

This feasibility study consists in determining the basic IVI signal features, the best IVI modality (single- or double-proton vertex imaging) and the influence on reconstructed vertex profiles of the main following parameters: ion range, target thickness and number of incident ions. The aim is threefold: (i) to evaluate the maximum target thickness beyond the Bragg peak allowing IVI monitoring (target-thickness influence), (ii) to determine the relation between the ion range and the reconstructed vertex distribution falloff (ion-range influence) and (iii) to 


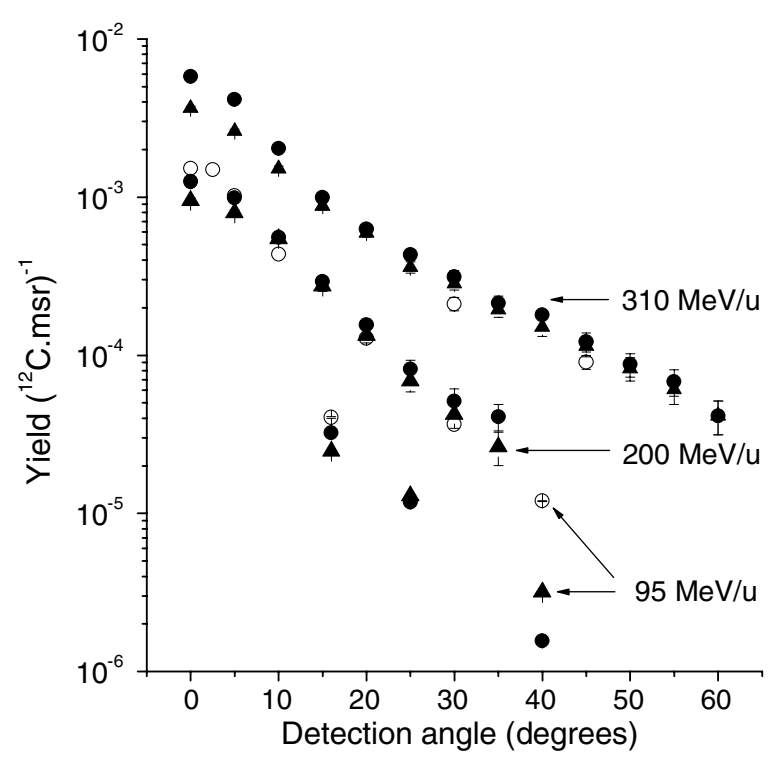

Figure 3. Experimental (open symbols) and simulated (filled symbols) proton emission yields as a function of emission angle and carbon ion energy (and target thickness): $310 \mathrm{MeV} \mathrm{u}^{-1}{ }^{12} \mathrm{C}$,

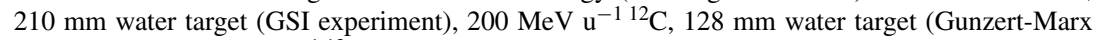
et al 2008) and $95 \mathrm{MeV} \mathrm{u}^{-112} \mathrm{C}, 25 \mathrm{~mm}$ PMMA target (Braunn et al 2011). Simulations have been performed with the QMD (circles) and BC (triangles) models.

give an order of magnitude of the number of incident ions required to measure the ion range with millimetric precision (influence of the number of incident ions).

In order to define the position of the distribution falloff, the vertex distribution is fitted with the following four-parameter sigmoid function: $a+b \times \operatorname{erfc}[c(z-d)]$, where $z$ is the target depth, erfc is the complementary error function and $a, b, c$ and $d$ are the parameters; $d$ corresponds to the inflection point of the complementary error function, which can be assumed to provide reliable information to estimate the ion range.

2.3.1. Basic IVI signal features. The basic IVI signal features are the generated vertex profile and the secondary proton features. We simulated a generated vertex profile for a carbon ion beam of intermediate energy: $200 \mathrm{MeV} \mathrm{u}^{-1}$ carbon ions incident on a cylindrical PMMA target of $10 \mathrm{~cm}$ in length and diameter. Regarding secondary proton features, they are well known through nuclear reaction studies, both for nuclear physics fundamental research (see, e.g., (Hüfner 1985, Lynch 1987)) and for hadrontherapy purpose (Schardt et al 2010). Some experiments aiming at measuring yields of secondary fragments were recently carried out at GANIL (95 MeV u ${ }^{-112} \mathrm{C}$ on thick-a few centimeters-targets in 2008 (Braunn et al 2011) and thin targets of various materials in 2011) and at GSI $\left(0.2-1 \mathrm{GeV} \mathrm{u}^{-1}\right.$ carbon, oxygen and iron ions on thin carbon, silicon and gold targets in August 2011). Data from Gunzert-Marx et al (2008) and Braunn et al (2011) have been used in this work.

It has been shown that fragments emitted from the projectile are produced with about the same velocity as the primary ions. As they have a lower charge than incident projectiles, their range is in general longer, which produces a dose tail after the Bragg peak. Among the charged fragments that escape from the target, protons constitute a large majority, which is the main motivation for focusing our discussion on proton properties. Moreover, the angular 


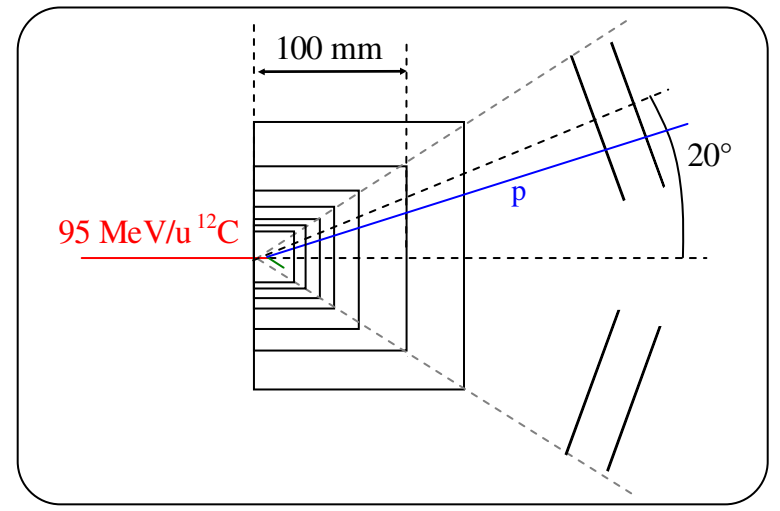

Figure 4. Diagram of the various vertex detection setups simulated to study the influence of the target thickness on the interaction vertex distributions for a ${ }^{12} \mathrm{C}$ ion beam with $2 \mathrm{~cm}$ range in PMMA. Cylindrical PMMA target thickness values: 25, 30, 40, 50, 70, 100 and $150 \mathrm{~mm}$.

distributions of fragments are relatively peaked in the beam direction due to nuclear reaction kinematics (Matsufuji et al 2005).

The main properties of secondary protons are partly illustrated in this work by simulated energy spectra and angular distributions of secondary protons emitted during nuclear reactions induced by ${ }^{12} \mathrm{C}$ ion beams $\left(75,150\right.$ and $\left.300 \mathrm{MeV} \mathrm{u}^{-1}\right)$ in a thin PMMA target ( $1 \mathrm{~mm}$ thick). Both $\mathrm{BC}$ and QMD results are presented in order to give an idea of model uncertainties. The differences between simulated and measured proton energy spectra emitted from a thick target (Gunzert-Marx et al 2008) are of the same order of magnitude.

2.3.2. Comparison of SP-IVI and DP-IVI. The comparison of single- and double-proton imaging is performed with the setup represented in figure 1 and $200 \mathrm{MeV} \mathrm{u}^{-1}$ incident carbon ions. The reconstructed vertex distributions are discussed in terms of statistics and shapes.

2.3.3. Target-thickness influence. As the falloff position of the vertex distribution is expected to be mainly driven by the secondary protons emitted typically in the last $20 \mathrm{~mm}$ of the carbon ion path, we investigate the target thickness issue with a low beam energy of $95 \mathrm{MeV} \mathrm{u}^{-1}$ (20 $\mathrm{mm}$ range in PMMA). The cylindrical PMMA target thickness values are the following: $25,30,40,50,70,100$ and $150 \mathrm{~mm}$ (figure 4). The target diameter varies in such a way that all detected protons escape from the target through the target exit surface perpendicular to the beam direction.

2.3.4. Ion-range influence. In order to study the influence of the ion range on the reconstructed vertex distributions, a clinically more relevant target is used: a head phantom consisting of a hollow bone-equivalent sphere (density $1.85 \mathrm{~g} \mathrm{~cm}^{-3}$ ) containing cortical tissueequivalent material (density $1.03 \mathrm{~g} \mathrm{~cm}^{-3}$ ). The sphere outer diameter and thickness are 200 and $3 \mathrm{~mm}$, respectively. The distance between the trackers and target entrance is increased by $5 \mathrm{~cm}$ with respect to the previous simulation geometry due to the larger target size (figure 5).

2.3.5. Influence of the number of incident ions. Finally, the statistical fluctuations of the vertex profile are likely to be the main source of uncertainties in the context of 'real-time' 


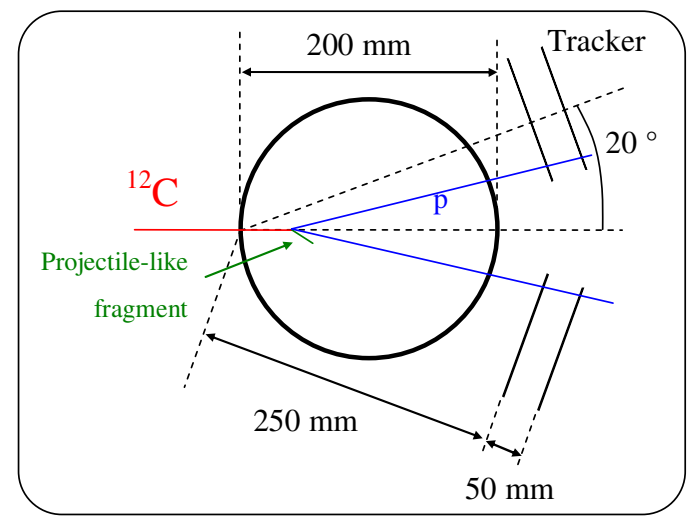

Figure 5. Diagram of the spherical head phantom simulation used to study the influence of the ion range on the interaction vertex distributions. It consists of a hollow bone-equivalent sphere filled with cortical tissue-equivalent material. The sphere diameter and thickness are 200 and $3 \mathrm{~mm}$, respectively.

ion-range monitoring. The monitoring of an active beam delivery system would ideally provide feedback for each raster position. About $7 \times 10^{8}{ }^{12} \mathrm{C}$ ions are needed to deliver an absorbed dose of $1 \mathrm{~Gy}$ in a tumor of $120 \mathrm{~cm}^{3}$ located between 6 and $11 \mathrm{~cm}$ in depth (Krämer et al 2000). With about $10^{4}$ raster positions, the number of incident ions $N_{\text {ions }}$ per raster position typically ranges from $10^{4}$ to $10^{6}$ (with higher $N_{\text {ions }}$ values at the distal edge).

\section{Results}

\subsection{Basic IVI signal features}

3.1.1. Generated vertex distributions. Figure 6 presents the simulated longitudinal distribution of vertices generated by $200 \mathrm{MeV} \mathrm{u}^{-1}$ carbon ions incident on a cylindrical PMMA target. The depth-dose distribution (gray line) is represented in order to illustrate the $75 \mathrm{~mm}$ range of such ions in PMMA with a density of $1.19 \mathrm{~g} \mathrm{~cm}^{-3}$. Vertices induced by primary (blue line) and secondary (red line) ions are also indicated.

We can see that, already for $200 \mathrm{MeV} \mathrm{u}^{-1}$ incident ions, vertices are mainly induced by secondaries. As expected, the best correlation between the vertex profile and the ion range is obtained with the primary vertices produced by incident ions. We can also note a slight decrease in the distribution of primary vertices due to the attenuation of the primary ion beam. The drop of the total vertex distribution at the Bragg peak position is finally not as dramatic as one might expect. If we define the contrast as the ratio of the vertex yield at the Bragg peak and a few millimeters beyond the Bragg peak, the contrast is about 1.6. Nevertheless, as we will see in section 3.2 , protons emitted off secondary vertices are unlikely to escape from the target. Secondary vertices are mainly induced by light particles such as neutrons and protons, which lead to the emission of low-energy protons issued from target nuclei.

3.1.2. Secondary proton features. Figure 7 presents simulated energy spectra and angular distributions of secondary protons emitted during nuclear reactions induced by ${ }^{12} \mathrm{C}$ ion beams $\left(75,150\right.$ and $\left.300 \mathrm{MeV} \mathrm{u}^{-1}\right)$ in a thin PMMA target ( $1 \mathrm{~mm}$ thick). As we can see in the energy spectra, a large proportion of secondary protons emitted from the projectile is moving with 


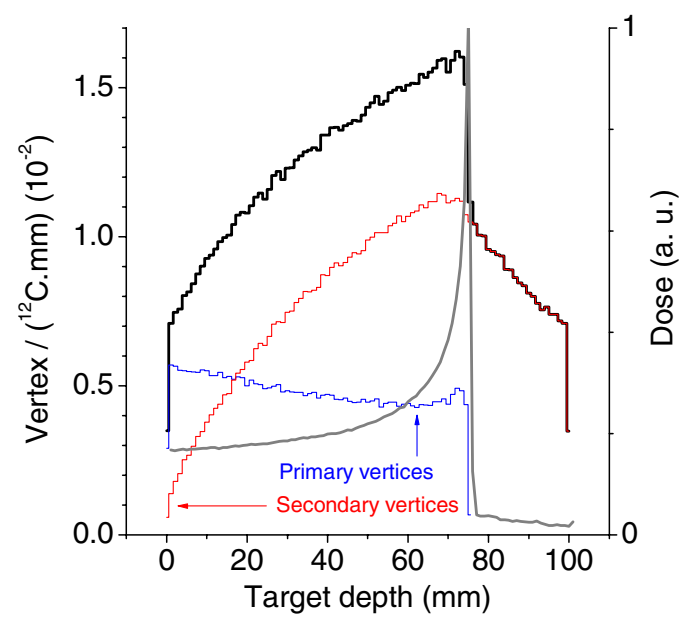

Figure 6. Longitudinal distribution of vertices generated in the simulations, for $200 \mathrm{MeV} \mathrm{u}^{-1}$ carbon ions incident on a cylindrical PMMA target of $10 \mathrm{~cm}$ in length and diameter. Secondary vertices correspond to vertices induced by secondary projectiles. The dose deposition profile is also represented (gray line).
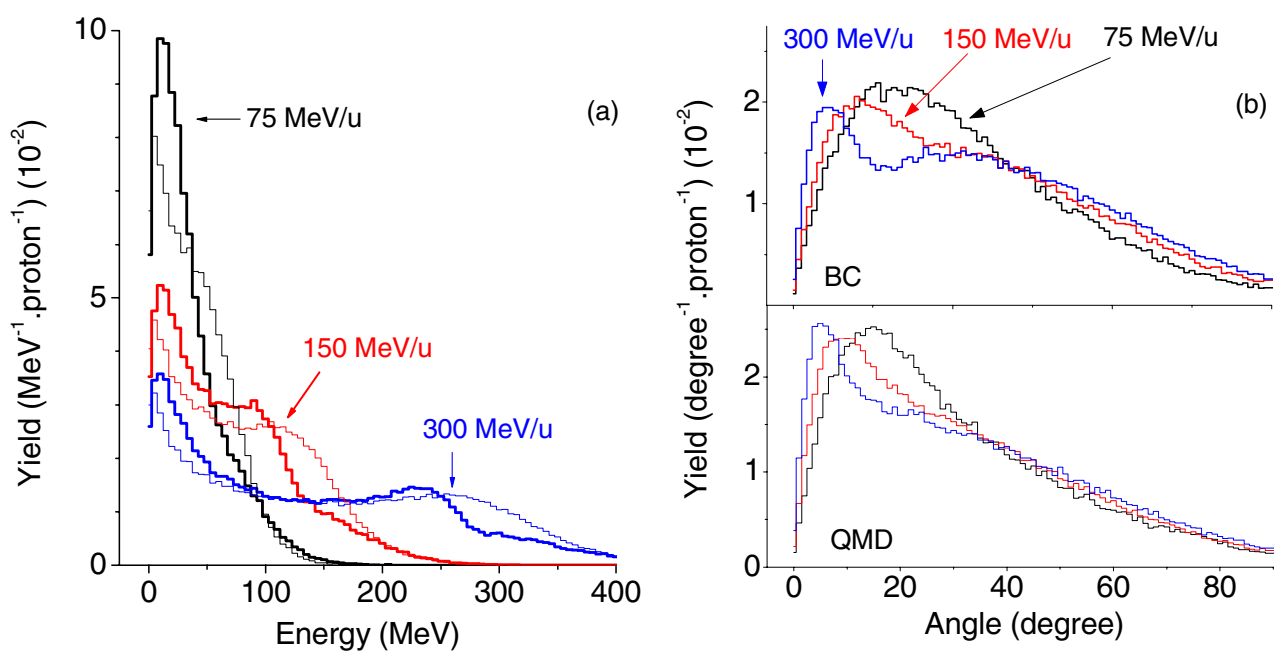

Figure 7. Energy spectra (a) and angular distributions (b) of secondary protons emitted from nuclear reaction vertices induced by ${ }^{12} \mathrm{C}$ ion beams $\left(75,150\right.$ and $\left.300 \mathrm{MeV} \mathrm{u}^{-1}\right)$ in a thin PMMA target (1 mm thick) (simulations using the BC (thick lines) and QMD models (thin lines)).

about the same velocity as the primary ions of 150 and $300 \mathrm{MeV} \mathrm{u}^{-1}$. At $75 \mathrm{MeV} \mathrm{u}^{-1}$ this component cannot be distinguished from the low-energy component due to protons emitted from target nuclei. Regarding angular distributions, we can observe that roughly half of the secondary protons are emitted at angles smaller than $40^{\circ}$, which provides a strong indication for positioning the trackers to maximize counting statistics. Energy and angular spectra obtained with the BC and QMD models are in overall good agreement.

Another crucial feature for double-proton imaging is the multiplicity distribution of protons emitted during nuclear reactions since this imaging technique consists in detecting 


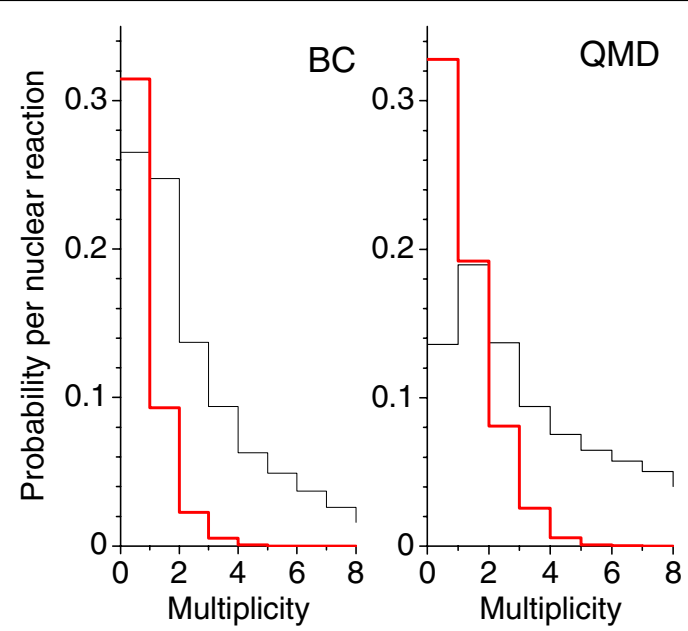

Figure 8. Multiplicity distributions of protons emitted during nuclear reactions induced by $300 \mathrm{MeV} \mathrm{u}^{-112} \mathrm{C}$ ion beams in a thin PMMA target (1 mm thick) with the BC (left) and QMD (right) models. Thin lines: total multiplicity distributions; thick lines: multiplicity distributions of protons with energies larger than $80 \%$ of the incident ion specific energy (in $\mathrm{MeV} \mathrm{u}^{-1}$ ). Note that a same event is likely to have its multiplicity reduced when the energy selection is applied, which explains why the first bin is higher.

two protons coming from the same interaction vertex. Figure 8 shows distributions obtained from $300 \mathrm{MeV} \mathrm{u}^{-112} \mathrm{C}$ ion beams with the $\mathrm{BC}$ and QMD models (thin black lines). Although these distributions are notably different, both of them have a relatively large mean multiplicity value between 3 and 4 . Nevertheless, in order to be fully meaningful for IVI, such multiplicity distributions have to be conditioned by relatively large proton energies in such a way that protons are likely to escape from the target. As the higher the primary ion energy, the longer its path in the target, we have chosen to define large proton energies as a percentage of the primary ion energy. The multiplicity distributions of protons with energies larger than $80 \%$ of the incident ion specific energy (in $\mathrm{MeV} \mathrm{u}^{-1}$ ) represented by thick red lines in figure 8 are dramatically reduced but the probability of multiple emissions remains promising around $15 \%$ (BC)-30\% (QMD) with $300 \mathrm{MeV} \mathrm{u}^{-112} \mathrm{C}$ ions and $10-20 \%$ with $75 \mathrm{MeV} \mathrm{u}^{-1}{ }^{12} \mathrm{C}$ ions (not shown). Double-proton imaging is therefore worthwhile to be investigated.

\subsection{Comparison of SP-IVI and DP-IVI}

Figure 9 shows the generated and reconstructed vertex distributions (solid lines) along with the generated positions of the reconstructed vertices (dotted lines), which will be denoted as 'real' vertex positions. Indeed, the reconstruction artifacts may lead to systematic deviations between generated and reconstructed positions. The single-proton distribution is one order of magnitude below the generated vertex distribution. This corresponds to a single-tracker setup efficiency of about $10 \%$, a fairly high value, which can be explained by the forward emission of protons.

The two-proton distribution is one order of magnitude lower than the single-proton one. This means that the two-proton detection probability roughly corresponds to the square of the single-proton detection probability. Given the large probability of multi-proton emission during one collision event (see figure 8), this is not surprising. Note that fortuitous events like the coincidence between two secondary protons emitted from two distinct vertices do not lead 


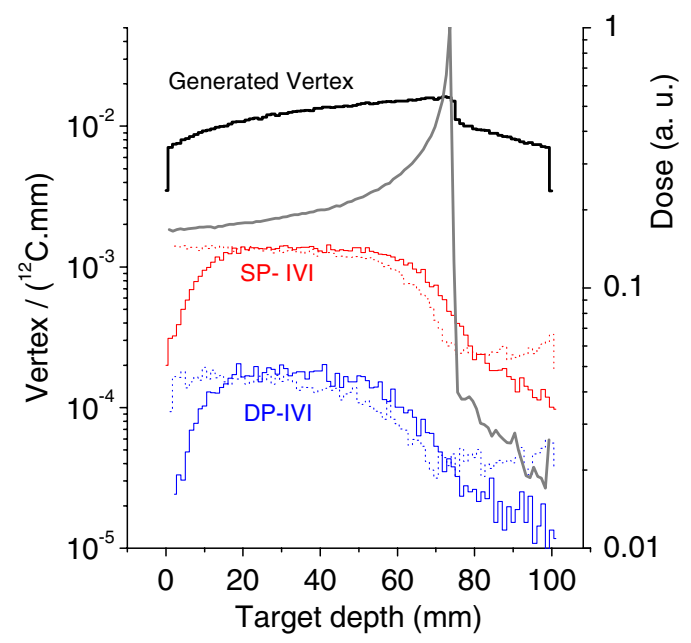

Figure 9. Total generated (top) and reconstructed (bottom) vertex yield distributions with the setup represented in figure 1 and $200 \mathrm{MeV} \mathrm{u}^{-1}$ incident carbon ions. Dotted lines correspond to the real positions of the reconstructed vertices. The depth-dose distribution is also shown. Number of incident ions: $10^{6}$.

to a dramatic deterioration of resolution. The shapes of the two distributions are very similar so that we can go right to the conclusion that single-proton detection is preferable, favoring high statistics, even if it requires a beam hodoscope to tag each incident carbon ion.

The comparison between the reconstructed and real vertex positions shows a shift of the falloff and a deficit in the reconstructed profile at target entrance and exit. Proton straggling in the target may lead to reconstructed trajectories relatively far from real vertices. In such cases, the segment corresponding to the minimum distance between the straight lines (proton and incident ion trajectories (SP-IVI) or the two-proton trajectories (DP-IVI)) tends to be downstream from the real vertex. This explains the shift between the reconstructed and real distribution drops and the reconstruction deficit at target entrance. The reconstruction deficit beyond the Bragg peak position corresponds to secondary vertices far from the beam position: the reconstruction assumption that vertices lie along the incident beam direction leads to reconstructed vertices upstream from the real vertices. Despite these differences between the real and reconstructed vertex profiles, the latter are strongly correlated with the ion range. Moreover their falloff contrast is about 5, much larger than the generated vertex distribution contrast. This confirms the expectation mentioned in the previous subsection: protons emitted from secondary vertices are unlikely to escape from the target. Finally, the detection statistics is high: for a beam intensity of the order of $10^{8}$ ions per second during carbon ion therapy, we can expect to reconstruct $10^{5}$ vertices per second per millimeter of the path of the primary beam with the aforementioned detection setup. IVI therefore seems promising for ion-range monitoring during carbon ion therapy.

\subsection{Target-thickness influence}

Figure 10 shows the reconstructed vertex distributions obtained with the various target thickness values, along with complementary error function fits. The reconstructed vertex yield clearly decreases as a function of target thickness since the escape probability of secondary protons decreases. The profile obtained with a $150 \mathrm{~mm}$ thick target is not represented because 

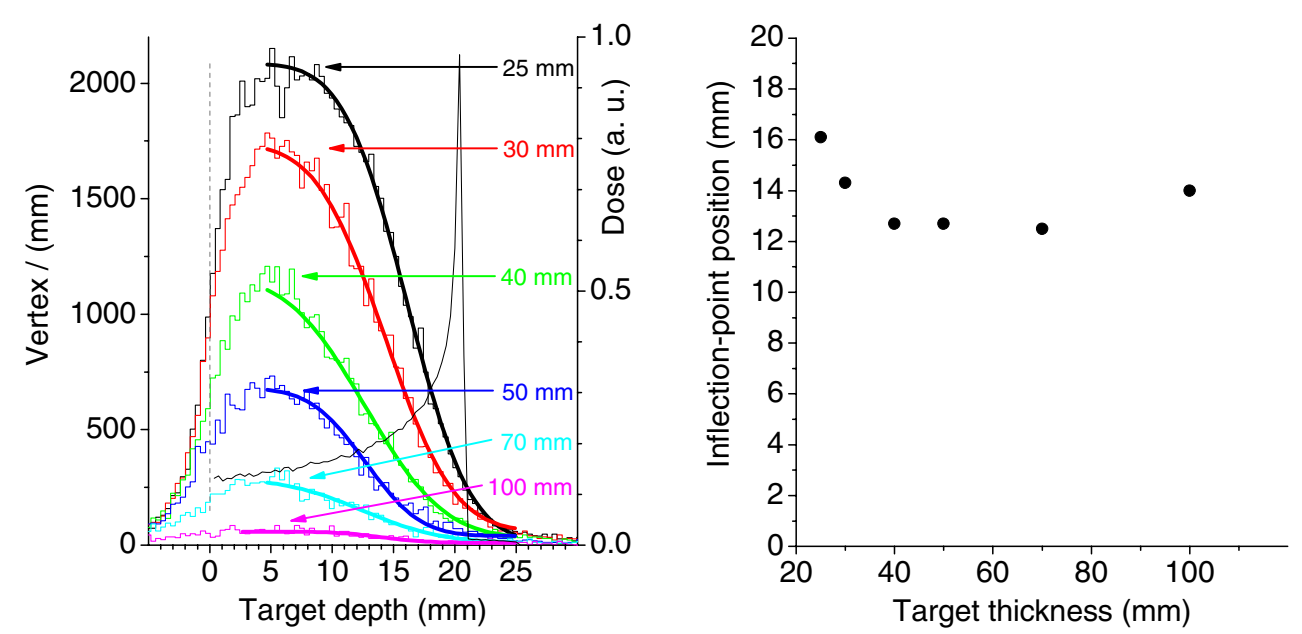

Figure 10. Left: SP-IVI reconstructed vertex distributions for a low beam energy of $95 \mathrm{MeV}$ $\mathrm{u}^{-1}$ ( $2 \mathrm{~cm}$ range in PMMA) and the various targets represented in figure 4 . The distribution obtained with a $150 \mathrm{~mm}$ thick target is not represented (it is flat with a yield of a few vertices $\mathrm{mm}^{-1}$ ). The smooth lines correspond to fits with the complementary error function. Right: Inflection point position (determined from the fits) as a function of the ion range. Number of incident ions per simulation: $10^{6}$.

only a few vertices are reconstructed per millimeter. A PMMA target thickness beyond the Bragg peak of about $10 \mathrm{~cm}$ would be the limit to perform IVI. We can also note a shift of the inflection point position $(d)$ from 16 to $13 \mathrm{~mm}$. The vertex distribution shape slightly changes when increasing the target thickness because the absorption of secondary protons affects more the low-energy protons emitted at the end of the ion path than those produced upstream. The increase of the inflection point position between the target thicknesses of 70 and $100 \mathrm{~mm}$ shows that the fit function is probably not appropriate in high attenuation conditions. The main conclusion that can be drawn from the previous observations is that IVI is able to provide information on the last two centimeters of the ion path for target thicknesses beyond the Bragg peak location lower than about $8 \mathrm{~cm}$. Further studies are considered to assess to what extent IVI can be sensitive to variations of the target density close to the Bragg peak.

It should be noted that, in principle, this target influence on the inflection point position is not an issue since the ion-range control consists in comparing the distributions of reconstructed vertices calculated in the treatment planning stage and measured during the irradiation. In practice, inter-fractional changes of the patient anatomy may have an influence on the reconstructed vertex distribution. Nevertheless, this influence should be much lower than a target thickness increase of $8 \mathrm{~cm}$ but it has to be studied further.

\subsection{Ion-range influence}

Figure 11(a) shows the reconstructed vertex distributions in the head phantom for various ion beam energies $\left(300,250,200\right.$ and $150 \mathrm{MeV} \mathrm{u}^{-1}$ ). As expected, the shorter the ion range, the lower the reconstructed vertex yields. It is noteworthy that the distribution obtained with the $150 \mathrm{MeV} \mathrm{u}^{-1}$ ion beam (blue line) is clearly visible, even though the target thickness beyond the Bragg peak is about $15 \mathrm{~cm}$ : with such a thickness beyond the Bragg peak, the reconstructed vertex distribution was not visible when using a $95 \mathrm{MeV} \mathrm{u}^{-1}$ ion beam. Indeed, apart from geometrical considerations (the path to escape the target was on average longer for the cylindrical target described above), the relatively high-energy protons emitted in the 

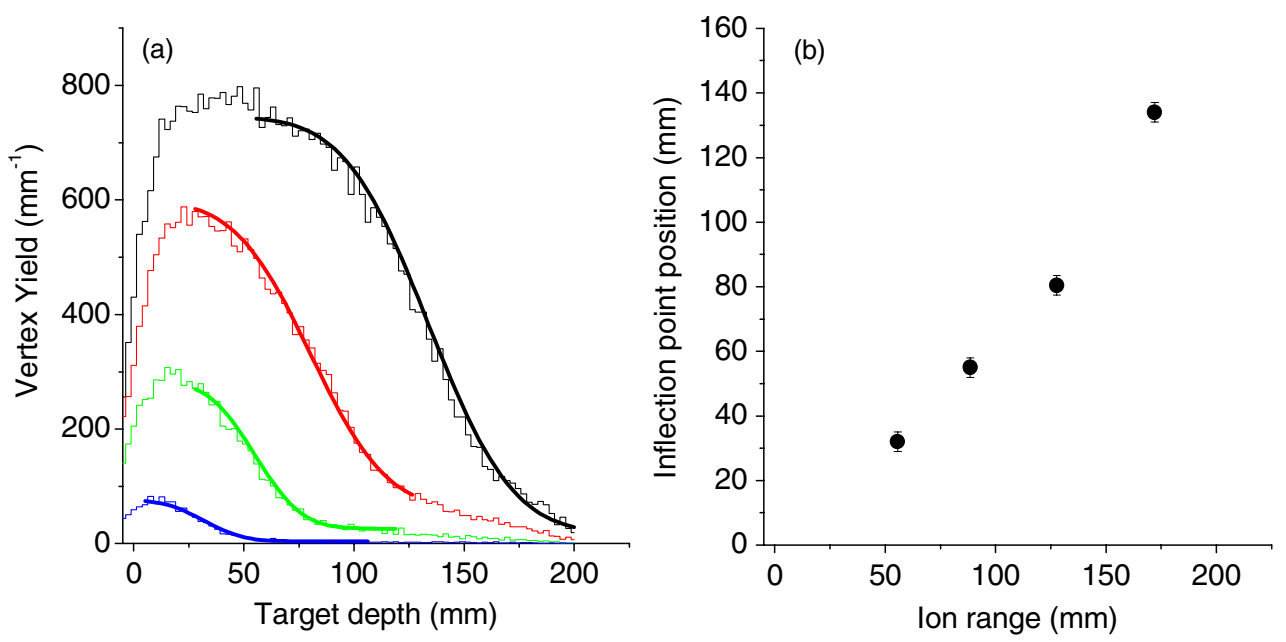

Figure 11. (a) SP-IVI reconstructed vertex distributions in the head phantom (diameter of $20 \mathrm{~cm}$ ) for various ion-beam energies $\left(300,250,200\right.$ and $\left.150 \mathrm{MeV} \mathrm{u}^{-1}\right)$. The smooth lines correspond to fits with the complementary error function. (b) Inflection point position (determined from the fits) as a function of the ion range. Number of incident ions per simulation: $10^{6}$.

first centimeters of the ion path are able to escape from the target and provide information on the ion range. Nevertheless, with such a target thickness $(15 \mathrm{~cm}$ beyond the Bragg peak), the current SP-IVI technique is unlikely to be very sensitive to any ion-range shift that may be induced by heterogeneities at the end of the ion path.

Figure 11(b) shows the inflection point position as a function of the ion range. This function is strictly increasing. At lower ion ranges, the influence of the target thickness beyond the Bragg peak is not negligible, which leads to a change of the function slope.

\subsection{Influence of the number of incident ions}

In order to evaluate the uncertainties in the vertex profile due to statistical fluctuations, a simulation with $10^{8}$ incident carbon ions of $200 \mathrm{MeV} \mathrm{u}^{-1}$ was carried out. This simulation was processed in order to vary the size of the incident ion bunches: from 5000 bunches of $2 \times 10^{4}$ ions to 100 bunches of $10^{6}$ ions. Each vertex profile was fitted with the complementary error function. Distributions of inflection point positions $d$ were calculated for $N_{\text {ions }}$ ranging from $2 \times 10^{4}$ to $2 \times 10^{6}$. The inset of figure 12 shows the distribution of $d$ values obtained for $N_{\text {ions }}=10^{5}$. The standard deviation $\sigma_{d}$ of the inflection point position distribution is presented as a function of $N_{\text {ions }}$ in figure 12. The line corresponds to the following fit function: $p_{1}+p_{2} / \sqrt{N_{\text {ions }}}$ with $p_{1}=0.7 \mathrm{~mm}$ and $p_{2}=356 \mathrm{~mm}$.ion ${ }^{1 / 2}$. As expected $\sigma_{d}$ presents a behavior close to an inverse square root law with a steep decrease up to $N_{\text {ions }} \sim 2 \times 10^{5}$. If $5 \mathrm{~mm}$ FWHM is considered as a reasonable confidence interval, we believe that IVI can provide reliable information with a number of incident ions $N_{\text {ions }}$ of the order of $10^{5}$ or even lower if the detection solid angle can be increased. This conclusion would remain valid even if we increase the ratio between simulated and measured secondary proton yields up to a factor of 2 .

\section{Conclusion}

In this work, we have investigated the feasibility of ion-range monitoring during carbon ion therapy by means of interaction vertex imaging with secondary protons. This feasibility study 


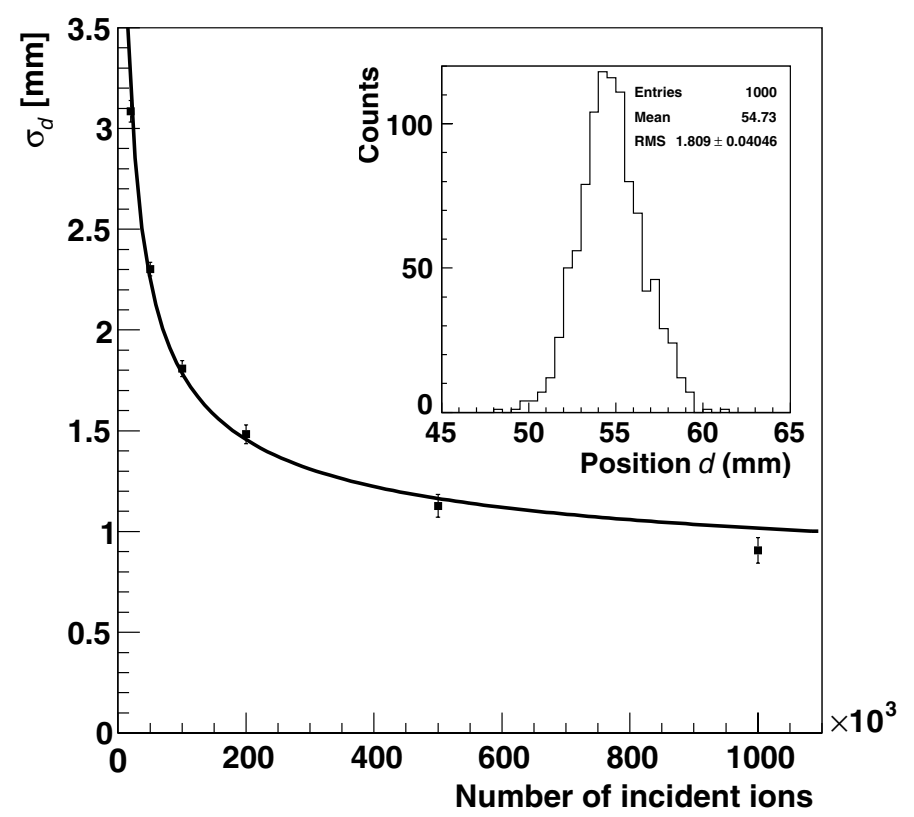

Figure 12. Standard deviation of the distribution of inflection point positions $d$ for various incident ion numbers. Simulation conditions: irradiation of the head phantom with a beam of $200 \mathrm{MeV} \mathrm{u}^{-1}$. The line corresponds to an inverse square root function (see the text). Inset: distribution of the inflection point positions for 1000 bunches of $10^{5}$ incident ions.

was performed with the Geant4 Monte Carlo simulation toolkit (version 9.2) validated against experimental data of secondary proton yields. The secondary proton yields obtained at GSI in the context of this feasibility study were reported.

The comparison of single- and double-proton interaction vertex imaging systems showed that the former is clearly the most promising, although it requires the development of a challenging beam hodoscope, under progress at IPNL. The target thickness and ion energy have a major influence on reconstructed vertex profiles. The main conclusion is probably that IVI is able to provide information on the last two centimeters of the ion path for (tissueequivalent) target thicknesses beyond the Bragg peak location lower than about $8 \mathrm{~cm}$.

Spatial resolution and detection efficiency are the main features of an imaging device. As already mentioned in the introduction, ion-range monitoring is based on the comparison of nuclear imaging distributions (PET, prompt gamma or interaction vertex imaging) calculated in the treatment planning stage (Monte Carlo simulations) and measured during the irradiation. In the case of interaction vertex imaging, uncertainties will be mainly due to heterogeneities at the end of the ion path (that may not affect the reconstructed vertex profile) and statistical fluctuations. Further studies are in progress to determine whether IVI can detect variations of target density at the end of the ion path. The influence of inter-fractional anatomical changes on reconstructed vertex distributions will be studied as well. Regarding statistical fluctuations, our feasibility study shows that monitoring every pencil beam during active treatment delivery should be possible: millimetric precision can be obtained with $2 \times 10^{5}$ incident ions, which is a typical number of ions per raster position.

This work is an ongoing effort, which gives rise to both simulation studies to optimize the system, and to experimental tests using the beam hodoscope developed at IPNL and the CMOS tracking detectors provided by IPHC-Strasbourg. 


\section{Acknowledgments}

This research project has been partly supported by the Regional Program for Research in Hadrontherapy (PRRH-ETOILE funded by the Rhône-Alpes Region) and the ENVISION European project (grant agreement no 241851). Monte Carlo simulations have been run at the IN2P3 Computing Center (CC-IN2P3) in Lyon, France.

\section{References}

Amaldi U, Hajdas W, Iliescu S, Malakhov N, Samarati J, Sauli F and Watts D 2010 Advanced quality assurance for CNAO Nucl. Instrum. Methods A 617 248-9

Apostolakis J et al 2009 Geometry and physics of the Geant4 toolkit for high and medium energy applications Radiat. Phys. Chem. 78 859-73

Baudot J et al 2009 First test results of MIMOSA-26, a fast CMOS sensor with integrated zero suppression and digitized output Nucl. Sci. Symp. Conf. Rec. (NSS/MIC) 2009 IEEE pp 1169-73

Böhlen T T, Cerutti F, Dosanjh M, Ferrari A, Gudowska I, Mairani A and Quesada J M 2010 Benchmarking nuclear models of FLUKA and GEANT4 for carbon ion therapy Phys. Med. Biol. 55 5833-47

Braunn B et al 2011 Nuclear reaction measurements of $95 \mathrm{MeV} \mathrm{u}^{-1} 12 \mathrm{C}$ interactions on PMMA for hadrontherapy Nucl. Instrum. Methods B $2692676-84$

Enghardt W, Crespo P, Fiedler F, Hinz R, Parodi K, Pawelke J and Pönisch F 2004 Charged hadron tumour therapy monitoring by means of PET Nucl. Instrum. Methods A $525284-8$

Fassò A, Ferrari A, Ranft J and Sala P R 2005 FLUKA: a multi-particle transport code CERN-2005-10, INFN/TC05/11 and SLAC-R-773

Grevillot L, Frisson T, Zahra N, Bertrand D, Stichelbaut F, Freud N and Sarrut D 2010 Optimization of GEANT4 settings for Proton Pencil Beam Scanning simulations using GATE Nucl. Instrum. Methods B 268 3295-305

Gudowska I, Sobolevsky N, Andreo P, Belkic D and Brahme A 2004 Ion beam transport in tissue-like media using the Monte Carlo code SHIELD-HIT Phys. Med. Biol. 49 1933-58

Gunzert-Marx K, Iwase H, Schardt D and Simon R S 2008 Secondary beam fragments produced by $200 \mathrm{MeV} \mathrm{u}^{-112} \mathrm{C}$ ions in water and their dose contributions in carbon ion radiotherapy New J. Phys. 10075003

Hüfner J 1985 Heavy fragments produced in proton-nucleus and nucleus-nucleus collisions at relativistic energies Phys. Rep. 125 129-85

Iwase H, Niita K and Nakamura T 2002 Development of general-purpose particle and heavy ion transport Monte Carlo code J. Nucl. Sci. Technol. 39 1142-51

Krämer M, Jäkel O, Haberer T, Kraft G, Schardt D and Weber U 2000 Treatment planning for heavy-ion radiotherapy: physical beam model and dose optimization Phys. Med. Biol. 45 3299-317

Lynch W G 1987 Nuclear fragmentation in proton- and heavy-ion-induced reactions Annu. Rev. Nucl. Sci. 37 $493-535$

Matsufuji N et al 2005 Spatial fragment distribution from a therapeutic pencil-like carbon beam in water Phys. Med. Biol. 50 3393-403

Min C-H, Kim C H, Youn M-Y and Kim J-W 2006 Prompt gamma measurements for locating the dose falloff region in the proton therapy Appl. Phys. Lett. 89 183517-3

Moteabbed M, España S and Paganetti H 2011 Monte Carlo patient study on the comparison of prompt gamma and PET imaging for range verification in proton therapy Phys. Med. Biol. 56 1063-82

Pshenichnov I, Botvina A, Mishustin I and Greiner W 2010 Nuclear fragmentation reactions in extended media studied with Geant4 toolkit Nucl. Instrum. Methods B 268 604-15

Pshenichnov I, Mishustin I and Greiner W 2008 Comparative study of depth-dose distributions for beams of light and heavy nuclei in tissue-like media Nucl. Instrum. Methods B 266 1094-8

Schardt D, Elsässer T and Schulz-Ertner D 2010 Heavy-ion tumor therapy: physical and radiobiological benefits Rev. Mod. Phys. 82383

Testa E et al 2008 Monitoring the Bragg peak location of $73 \mathrm{MeV} \mathrm{u}^{-1}$ carbon ions by means of prompt $\gamma$-ray measurements Appl. Phys. Lett. 93093506

Testa M et al 2010 Real-time monitoring of the Bragg-peak position in ion therapy by means of single photon detection Radiat. Environ. Biophys. 49 337-43

Villagrasa C, Francis Z and Incerti S 2011 Physical models implemented in the GEANT4-DNA extension of the GEANT-4 toolkit for calculating initial radiation damage at the molecular level Radiat. Prot. Dosim. 143 214-8

Ziegler J F, Ziegler M D and Biersack J P 2010 SRIM - the stopping and range of ions in matter (2010) Nucl. Instrum. Methods B 268 1818-23 